\title{
Targets, attitudes, and goals of psychiatrists treating patients with schizophrenia: key outcome drivers, role of quality of life, and place of long- acting antipsychotics
}

\author{
This article was published in the following Dove Press journal: \\ Neuropsychiatric Disease and Treatment \\ II January 2016 \\ Number of times this article has been viewed
}

\author{
Andrea de Bartolomeis' \\ Andrea Fagiolini ${ }^{2}$ \\ Marco Vaggi ${ }^{3}$ \\ Claudio Vampini ${ }^{4}$ \\ 'Section of Psychiatry and Treatment \\ Resistant Psychosis, Department of \\ Neuroscience, University of Naples \\ Federico II, Naples, Italy; ${ }^{2}$ Department \\ of Molecular and Developmental \\ Medicine, School of Medicine, \\ University of Siena, Siena, Italy; \\ ${ }^{3}$ Mental Health and Drug Addiction \\ Department, Genovese, Genoa, \\ Italy; ${ }^{4}$ Department of Mental Health, \\ Ospedale Civile Maggiore and ULSS 20, \\ Verona, Italy
}

Purpose: This survey of Italian psychiatrists was conducted to better define drivers of schizophrenia treatment choice in real-life practice, particularly for use of long-acting injectable (LAI) antipsychotics.

Methods: Between October 15 and December 15, 2014, 1,000 surveys were sent to psychiatrists who treat schizophrenic patients; 709 completed questionnaires were analyzed ( $71 \%$ response rate).

Results: The two most important factors determining therapy success were efficacy $(75 \%$ of responses) and tolerability (45\%) followed by global functioning (24\%) and quality of life (17\%). LAI antipsychotics were most often used to facilitate regular treatment monitoring (49\%), and $41 \%$ of psychiatrists thought that patients with low adherence who had failed oral therapy were well-suited for LAI antipsychotics. Only $4 \%$ of respondents saw LAI antipsychotics as appropriate for patients without other therapeutic options.

Conclusion: Although efficacy and tolerability were the most common factors used to evaluate treatment success in schizophrenia, psychiatrists also consider QoL and global functioning to be important.

Keywords: quality of life, long-acting injectable antipsychotics, schizophrenia, survey

\section{Objective}

Schizophrenia is a chronic, disabling, psychiatric disorder that is characterized by a variety of cognitive and emotional abnormalities. ${ }^{1}$ These symptoms and the general decline in functioning associated with schizophrenia ${ }^{1,2}$ mean that patients' everyday behavior and quality of life (QoL) are negatively affected. The mainstay of schizophrenia treatment is antipsychotic drugs. ${ }^{3,4}$ In accordance with current guidelines, the specific goals and outcomes of schizophrenia treatment vary according to the phase and severity of the condition. ${ }^{5-7}$ In the acute phase, the main target of pharmacological therapy is to reduce psychotic symptoms, while in the stabilization phase, which may last for several months, treatment is consolidated to minimize the likelihood of a relapse. ${ }^{5,8}$ This is followed by the stable phase (a prolonged period of treatment lasting several months to years), during which one of the primary aims is to continue recovery and improve functioning and QoL. ${ }^{5,8}$ However, the pathology of schizophrenia is such that it can have a negative impact on QoL before the long-term goals of treatment are reached.
Correspondence: Andrea Fagiolin Department of Molecular and

Developmental Medicine, University of Siena, Viale Bracci I, 53 100 Siena, Italy Tel +390577586275

Fax +390577233 451

Email andreafagiolini@gmail.com 
Functional disability in patients with schizophrenia is measured in terms of impairment in everyday living skills (eg, independent living skills, social functioning, vocational functioning, and self-care), ${ }^{9}$ while QoL is a holistic concept that includes assessment of physical and mental health (ie, health-related QoL [HRQoL]), social characteristics, and economic factors. ${ }^{10}$ Specifically, QoL is defined as an individual's perception of their position in life in the context of the culture and value systems in which they live and in relation to their goals, expectations, standards, and concerns. ${ }^{11} \mathrm{HRQ}$ L refers to the functional effect of a medical condition and/or its consequent therapy upon a patient; ${ }^{12,13}$ it is subjective and multidimensional, encompassing physical and occupational function, psychological state, social interaction, and somatic sensation. ${ }^{13}$ An individual's ability to perform the daily activities required to meet basic needs, fulfill usual roles, and maintain health and well-being is described as "functioning". ${ }^{14}$

Functioning and QoL can be influenced by a variety of factors, including those related to disease pathology (eg, the depressive and negative symptoms associated with schizophrenia) and factors related to pharmacological treatment (eg, drug dosage and efficacy, duration of treatment, and subjective tolerability). ${ }^{15-17}$ Treatment benefits with antipsychotics have been assessed both in efficacy trials (evaluating the performance of antipsychotics in the controlled conditions of a clinical trial) and in studies assessing the effectiveness of agents in real-world settings. Evaluation of effectiveness is broader than assessment of efficacy, and takes into account the effects of treatment on symptoms and potential side effects as well as the effects of treatment on a patient's everyday functioning and well-being, family burden, and treatment adherence. ${ }^{18}$ Real-world studies in schizophrenia have utilized several scales to assess improvements in QoL, including the Quality of Life Scale (QLS), ${ }^{19}$ Short-Form Health Survey 36 (SF-36), ${ }^{20}$ Tolerability and Quality of Life (TOOL), ${ }^{21}$ Subjective Well-being (SWN) scale, ${ }^{22}$ RiedelSpellmann-Musil scale, ${ }^{23}$ and World Health Organization Quality of Life Scale, Brief Version (WHOQOL-BREF). ${ }^{24}$

Most patients with schizophrenia require long-term maintenance treatment to control their symptoms, but despite the availability of several effective antipsychotics, many do not receive adequate long-term therapy, mainly due to poor adherence. ${ }^{25-28}$ Clinical studies have shown that optimizing the balance between efficacy and side effects is very important for improving adherence and maximizing QoL. ${ }^{6,29,30}$ Studies have also highlighted the need to target nonpsychotic symptoms of schizophrenia and manage social and economic factors to maximize QoL. ${ }^{15,31}$
Antipsychotics may impact differently on QoL, with studies suggesting that atypical antipsychotics may have a more positive impact on QoL than typical antipsychotics, possibly because of a slightly favorable effect on negative symptoms and better subjective tolerability. ${ }^{32-34}$ In addition, the mode of administration, frequency, and ease of use all play a major role in the overall effectiveness of antipsychotic therapy ${ }^{35}$ A recent time trade-off study showed that longer injection intervals were associated with improved HRQoL (utility), suggesting that the use of long-acting injectable (LAI) antipsychotics may improve adherence, leading to improved QoL. ${ }^{36}$

Data from clinical trials suggest that measurement of treatment success should include assessment of psychosocial, treatment-related, and health care system-related factors. ${ }^{37-40}$ However, studies investigating psychiatrist opinion on the most important factors for evaluating success of antipsychotic therapy for schizophrenia in the clinic setting are lacking. The objective of this survey was to identify the primary drivers for treatment choice and the variables by which psychiatrists evaluate the success of antipsychotic therapy in general, and LAI in particular, in patients with schizophrenia.

\section{Materials and methods Questionnaire development}

The study comprised an extensive survey of Italian psychiatrists chosen on the basis of their expertise in treating patients affected by schizophrenia and representative of different mental health settings (community hospital, mental health centers and other community-based institutions, private practice, rehabilitation). The selection of participants was based on their profile and experience in treating patients with schizophrenia, including those receiving LAI antipsychotics. The questionnaire was developed by a scientific board of four Italian psychiatrists, and the survey was conducted by an external worldwide society focused on market research and surveys in the pharmaceutical field (Cegedim Strategic Data).

The questionnaire (see Supplementary Material for full questionnaire) comprised a series of questions structured in sections to gather opinions about the following issues regarding treatment of patients with schizophrenia:

1. Which parameters are used by specialists to evaluate the success of an antipsychotic treatment?

2. How do psychiatrists define and assess QoL?

3. Which scales are considered the most useful for the assessment of QoL?

4. How important is QoL measurement as an outcome to be investigated in a clinical trial? 
5. Which therapeutic goals are achievable with LAI antipsychotic therapy?

6. Which types of patients should be prescribed LAI antipsychotic therapy?

The questionnaire was a self-rated instrument, comprising open-ended questions with free text responses, questions with binary (Yes/No) responses, and multiple-choice questions assessing objective facts and subjective states. Unsolicited, multiple-choice test items were used to measure the understanding and the application of knowledge and skills to complex situations, such as critical thinking and problem solving. In this case, multiple answers could be selected. The survey also included a multiple-choice question about which scales were considered the most useful for the evaluation of QoL in patients with schizophrenia.

Each section of the questionnaire included items organized into facets, including objective and subjective domains. When answers required the respondent to express relative and subjective preference or agreement, a 1- to 7-point rating scale was used, with 1 representing the lowest importance/ preference/or complete disagreement, while 7 was equivalent to the utmost importance/highly preferred/or complete agreement. Written informed consent for participants and confidentiality on data managing were stated in the questionnaire, in accordance with the Data Protection Act of the Italian Law. Institutional Review Board approval was not deemed necessary for the following study. All principles outlined in the Declaration of Helsinki were followed.

\section{Survey conduct}

The survey was conducted between October 15 and December 15, 2014. Questionnaires were available in Italian only, and were sent out to 1,000 psychiatrists equally distributed throughout Italy. The questionnaires were selfcompleted by the participants and were anonymized (to try and increase the rate of response).

\section{Statistical analysis}

A large sample was selected in order to achieve the lowest possible margin of error compatible with the feasibility of the study. A sample of approximately 700 psychiatrists from the approximately 10,000 practicing in Italy was determined to reduce the statistical error to $3.5 \%$ (ie, range of error $\pm 3.5 \%$ ); the error rate was not chosen a priori, but calculated taking into account the sample number of psychiatrists participating to the survey (709) and the total population of Italian psychiatrists $(8,000)$. A sample number of 709 psychiatrists has been chosen based on the feasibility of the study, in terms of timing and costs. The survey was sent out to 1,000 psychiatrists because the likely response rate was estimated as $70 \%$.

All analyses were conducted by Cegedim Strategic Data and were performed using $\mathrm{SAS}^{\circledR}$ software version 9.4 (SAS Institute Inc, Cary, NC, USA). Responses were analyzed as a percentage of the total number of times the component was selected. Dichotomous variables were reported as a percentage of survey participants.

The questions regarding parameters considered by Italian psychiatrists to be the most important when evaluating the success of an antipsychotic therapy in patients with schizophrenia were formulated as both unsolicited, open-ended questions and multiple-choice answers, leaving the possibility of selecting more than one composite parameter. For questions requiring relative and subjective importance or agreement, a 7-point scale was used instead of a 10-point scale to increase confidence in subjective answers and to avoid influence of personal characteristics and psychological bias, ie, answer error, with optimistic responders tending to overrate and pessimistic responders tending to underrate.

Qualitative variables were described using established statistical methodologies, using frequencies and percentages, while quantitative variables were reported as mean, standard deviation, median, and minimum and maximum values.

\section{Results}

\section{Survey respondents}

Of the 1,000 surveys sent out to psychiatrists in Italy, 750 completed surveys were received, of which 41 could not be analyzed because they were incomplete in one or more of the major items, leaving 709 completed surveys for analysis ( $71 \%$ response rate). Of these, 145 came from the Northwest of Italy, 57 from the Northeast, 133 from the Central Region, 175 from the South of Italy and from the Islands (Sicily and Sardinia), while 199 did not report any location.

The respondents had a mean professional experience of 17 years and were mainly psychiatry specialists (98\%); the characteristics and professional profile of the respondents are presented in Table 1. The distribution of the overall population in terms of work environment was similar to the distribution seen when respondents were analyzed according to region (Figure 1). The mean number of patients with schizophrenia seen per institution per year was 237.1 (median: 148.3). During the month preceding the survey, a mean of 72.6 (median 39.4) patients with schizophrenia visited the institution; among these, the respondents were in charge of a mean 21.8 (median: 22.6) patients. 
Table I Characteristics of the overall population of survey respondents

\begin{tabular}{ll}
\hline Characteristics & $\begin{array}{l}\text { Percentage of } \\
\text { respondents }\end{array}$ \\
\hline Sex & 38 \\
Male & 25 \\
Female & 37 \\
$\quad$ Not specified & \\
Place of work & 60 \\
$\quad$ Community mental health centers/community- & \\
based institutions & 27 \\
Inpatient hospitals & 8 \\
Private practice & 5 \\
Rehabilitation setting & \\
Years of professional experience & 14 \\
$\leq 10$ years & 15 \\
II-20 years & 16 \\
$2 I-30$ years & 2 \\
$>30$ years & 53 \\
No response & \\
\hline
\end{tabular}

\section{Parameters for evaluating success of antipsychotic therapy in patients with schizophrenia}

The composite parameter "efficacy" was reported most often, followed by "tolerability/safety" and "global functioning" (Figure 2). Within the composite parameter of efficacy, the component "efficacy/optimization of therapeutic response" was chosen in $32 \%$ of the answers, "effective on positive/ productive symptoms" in $25 \%$, "control/improvement of symptoms" in $16 \%$, and "effective on negative symptoms" in $12 \%$. Long-term efficacy was mentioned by only $3 \%$ of respondents, and only $5 \%$ linked efficacy to relapse prevention. The clinician's perception of "global functioning" was primarily based on the components of "participation to social life/social functioning" (11\% of total answers), followed by "functioning" and "improvement of personal relationships" (14\% of total

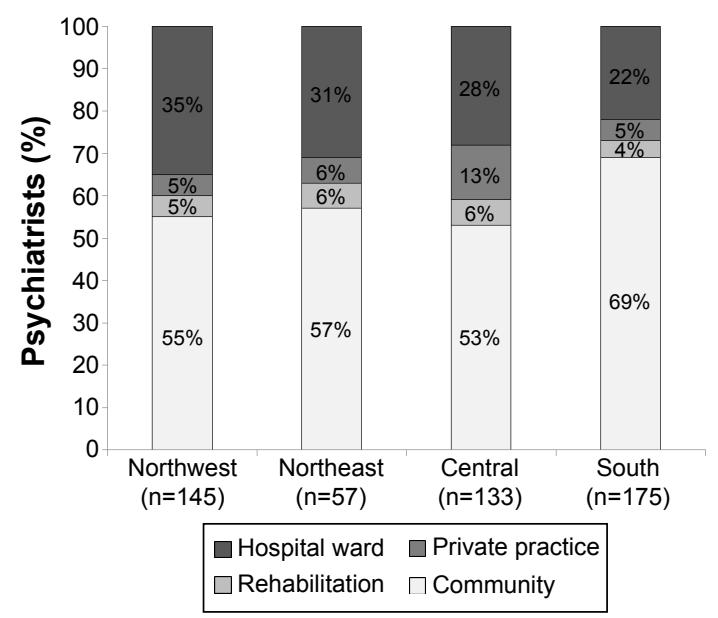

Figure I Regional distribution of respondents according to mental health settings where the respondents performed professional activities.

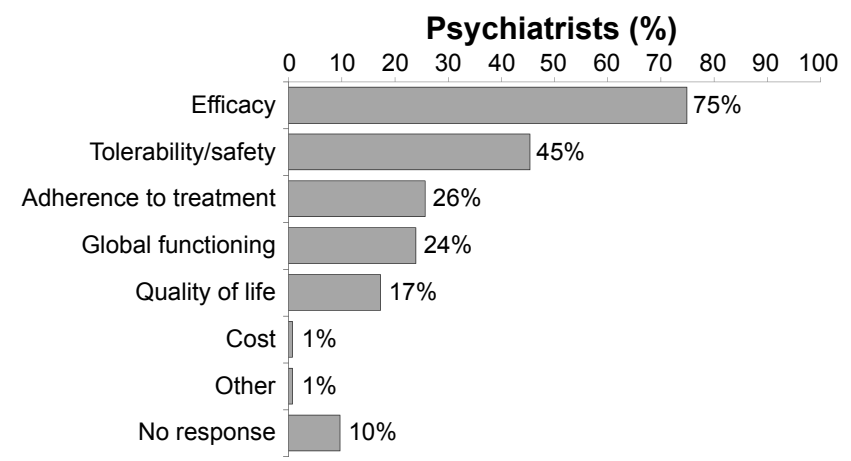

Figure 2 Suggested parameters to be considered when evaluating the success of an antipsychotic therapy in patients with schizophrenia.

Notes: Responses to the question "Which parameters do you consider when evaluating the success of antipsychotic therapy in patients suffering from schizophrenia?" Percentage of the total number of times the specific domain has been reported in answering this open-ended unsolicited question by the 709 respondents.

answers), while "cognitive improvement" and "work/school performance" (4\% of total answers each) were less valued.

QoL was mainly identified as "improvement in QoL", accounting for $14 \%$ of the total answers. When respondents were asked what other factors besides therapeutic efficacy indicated treatment success using preset domains and the 7-point scale, where 1 was "not important at all" and 7 was "of the utmost importance", "adherence to treatment", and "QoL achieved" were the most highly rated domains, with $59 \%$ and $47 \%$ of respondents scoring 7 , respectively (Figure 3). Overall mean scores for all the factors evaluating treatment success ranged from 6.1 (tolerability) to 6.4 (adherence to treatment).

\section{How Italian psychiatrists define QoL in patients suffering from schizophrenia}

In response to the question "What do you mean by 'QoL' in patients suffering from schizophrenia?", $58 \%$ of respondents

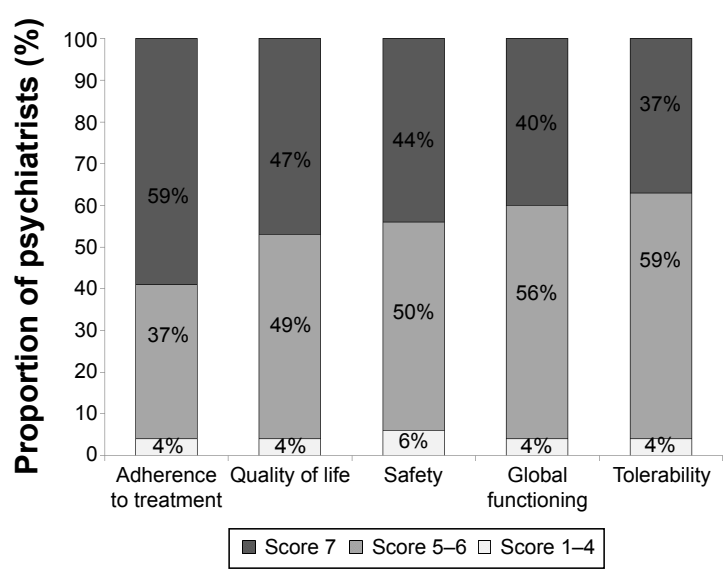

Figure 3 Domains of importance of preset responses when assessing treatment success rated from I (not important) to 7 (of utmost importance) by psychiatrists treating patients suffering from schizophrenia.

Notes: Responses to the question "How important do you think are the following items, except for the therapeutic efficacy, when assessing the success of an antipsychotic therapy in a patient with schizophrenia?" Percentage of responses mentioning the specific domain from a cohort of 709 respondents. 


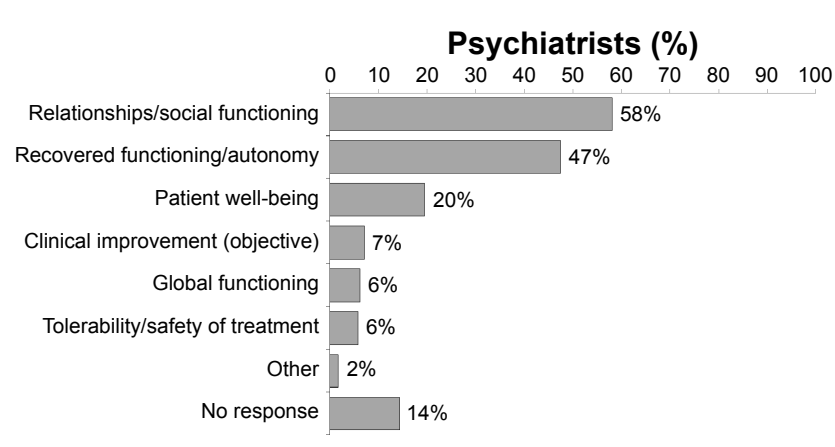

Figure 4 Psychiatrists' understanding of quality of life (QoL) in patients with schizophrenia.

Notes: Responses to the question "What do you mean by 'QoL' in a patient suffering from schizophrenia?" Percentage of responses mentioning the specific domain when clinicians were questioned about the meaning of QoL from a cohort of 709 clinicians.

perceived QoL as "relationships/social functioning", $47 \%$ as "recovered functioning/autonomy", and $20 \%$ as "patient well-being" (Figure 4). The factor "good school/work performance" was cited as part of QoL by $26 \%$ of psychiatrists surveyed and "objective clinical improvement" was mentioned at least once in $7 \%$ of the responses.

When respondents were asked to define QoL using preset domains and the 7-point scale, the factor most frequently assigned a score of 7 (of utmost importance) was "interpersonal relationship" ( $41 \%$ of respondents; Figure 5). Overall mean scores were similar across all aspects of QoL assessed, ranging from 5.8 for work/school performance to 6.2 for interpersonal relationships.

\section{Knowledge of scales to assess QoL}

Results of the multiple-choice question regarding QoL scales considered most useful for QoL evaluation in patients with schizophrenia showed that QLS was mentioned at least once in

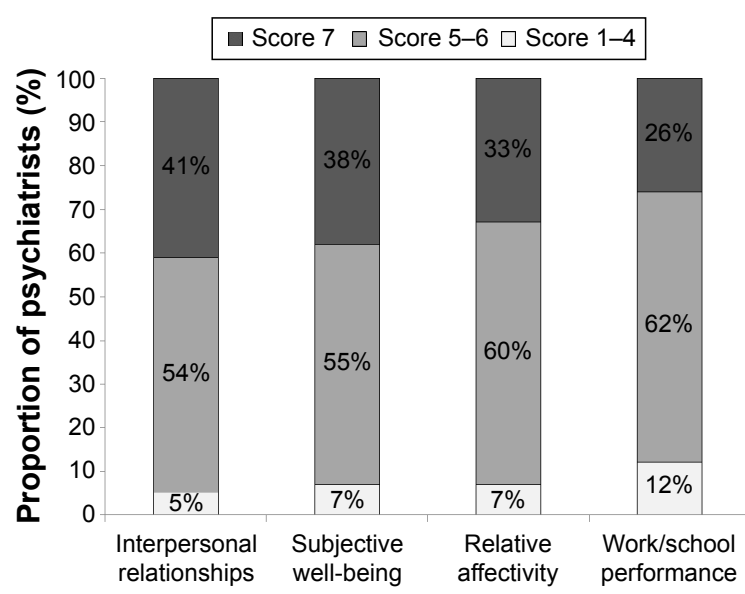

Figure 5 Importance of preset items when reporting understanding of quality of life (QoL) according to psychiatrists, rated from I (not important) to 7 (of utmost importance).

Notes: Responses to the question "Which are the most important items through which you assess the QoL in patients suffering from schizophrenia?" Percentage of responses mentioning the specific domain from a cohort of 709 respondents.
$67 \%$ of responses, SF-36 in 20\%, and TOOL in $15 \%$. However, a minority (3\%) of the surveyed specialists responded that they would not use any scale, while $12 \%$ left this section blank.

To the dichotomous question "Are you familiar with the QLS?", 76\% of the participants responded "Yes". When these respondents were asked if QLS could become a useful instrument in daily clinical practice, $87 \%$ of them provided a favorable response. Of the $13 \%$ of participants who responded negatively to this question, $26 \%$ stated that they had no time to use QLS in real-life practice.

\section{Importance of QoL assessment in clinical trials on treatment of schizophrenia}

To the question "In clinical trials on schizophrenia how important is the evaluation of patient's QoL?", 30\% of those surveyed responded that it was of utmost importance (score 7). However, it is noteworthy that $88 \%$ of them considered QoL assessment to be an important parameter for investigation in clinical trials, scoring from 5 to 7.

\section{Therapeutic targets achievable with LAI antipsychotics}

Overall, $97 \%$ of the psychiatrists surveyed said that they used LAI antipsychotics. Evaluating the importance of therapeutic targets achievable with LAI antipsychotic therapy using preset domains and the 7-point scale, "adherence" and "safety" were the most highly rated domains, with $52 \%$ and $47 \%$ of respondents, respectively, recording scores of 7 . Of note, $96 \%$ of specialists considered "QoL" to be an important goal (score 5 to 7 ), with $40 \%$ of them rating it as having the utmost importance (score 7) (Figure 6).

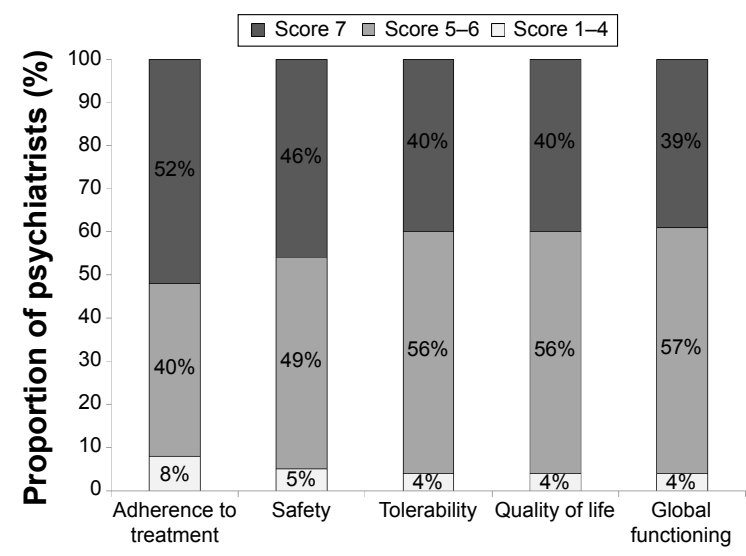

Figure $\mathbf{6}$ Level of importance of preset clinical outcomes when assessing the success of long-acting injectable treatment, rated from I (not important) to 7 (of utmost importance) according to psychiatrists treating patients with schizophrenia.

Notes: Responses to the question "How much importance do you attach to the following clinical outcomes (not including therapeutic efficacy) when assessing the success of long-acting antipsychotic therapy in a patient suffering from schizophrenia?" Percentage of responses mentioning the specific clinical outcomes from a cohort of 709 respondents. 


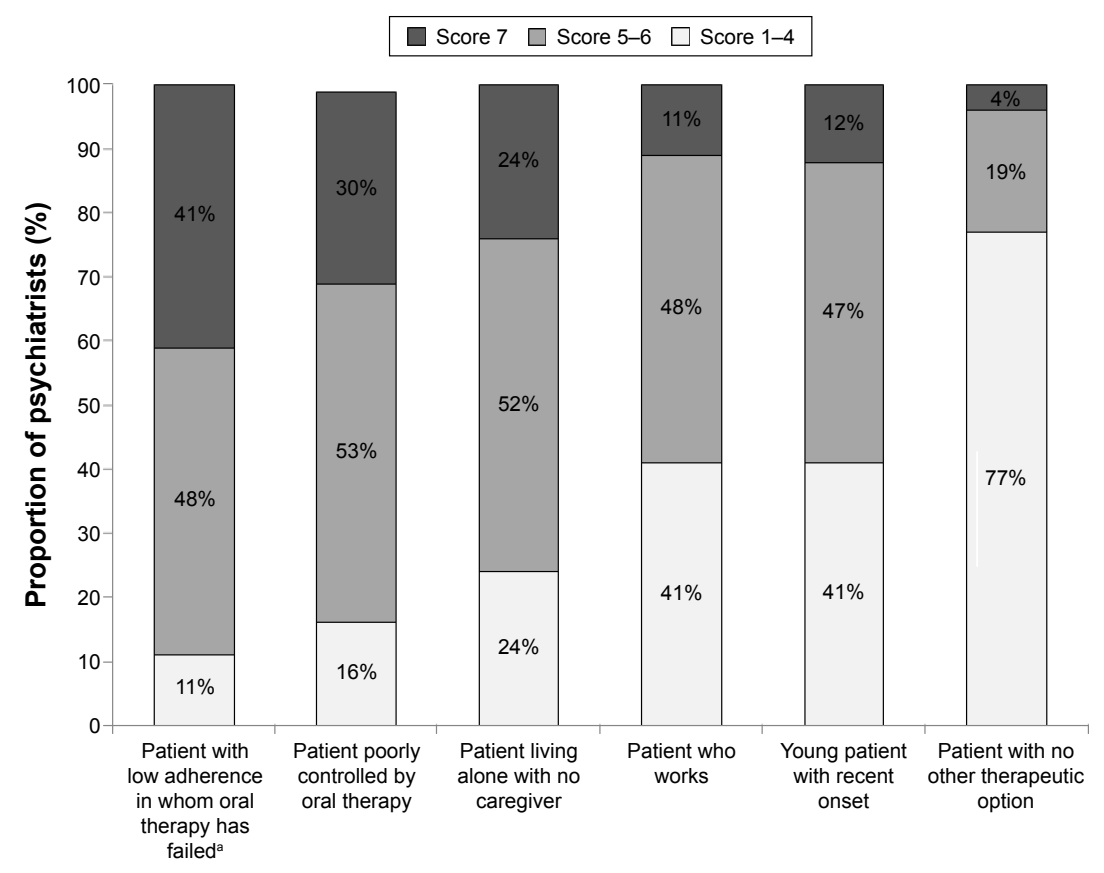

Figure 7 Level of agreement with prescribing long-acting injectable antipsychotic treatment to five preset patient types, rated from I (no agreement) to 7 (complete agreement) according to psychiatrists treating patients with schizophrenia.

Notes: Responses to the statement "These patients with schizophrenia are best suited to receiving long-acting antipsychotic therapy". Percentage of responses mentioning the specific patient type from a cohort of 709 respondents. ${ }^{2}$ Treatment failed in these patients due to a lack of patient compliance/not taking medicine.

Questions were raised about the expectations and objectives of treatment with LAI antipsychotics, based on multiple-choice answers and using the 7-point scale. Fortynine percent of psychiatrists ranked the statement that LAI antipsychotics "allow for regular monitoring of treatment administration" of utmost importance (a score of 7), while $40 \%, 20 \%, 17 \%$, and $14 \%$ of psychiatrists ranked the statements that LAI antipsychotics "allow better adherence to the therapeutic plan", "are treatment options to consider in the initial phase of treatment", "enable patients to become more independent", and "substantially improve patient QoL" of utmost importance.

\section{Types of patients better suited to LAI antipsychotics}

Evaluating the types of patients better suited to receive LAI antipsychotic treatment using six preset "patient types" and the 7-point scale, clinicians were asked to score their agreement for each patient type with the following statement: "These patients with schizophrenia are better suited to receive LAI therapy" with a score of 1 indicating no agreement and a score of 7 indicating complete agreement. Results showed that "patients with low adherence in which oral therapy has failed" had the highest mean score of 6.0, and $41 \%$ of psychiatrists scored this criterion a 7 , indicating that these patients were considered to be well-suited to
LAI antipsychotic therapy (Figure 7). This was followed by "patients poorly controlled on oral therapy" (mean: 5.7), and "patients living alone" (mean: 5.4; Figure 7). Only 4\% of specialists scored "patients with no other therapeutic option" as 7 , and $77 \%$ gave this item a score of $1-4$ (mean score: 3.0 ), indicating that the majority did not consider this patient group to be well-suited to LAI antipsychotic therapy. Most psychiatrists (86\%) said that they would change (increase) their use of LAI antipsychotics if the results of a clinical trial showed that an LAI antipsychotic could improve a patient's QoL.

\section{Discussion}

The 709 psychiatrists who completed the questionnaire were from all over Italy, covering a cross-section of psychiatric services and having a considerable depth of experience in terms of duration and number of schizophrenia patients treated. The treatment behavior they reported was in accordance with international guidelines. According to these psychiatrists, the most common factor influencing success of antipsychotic therapy in patients with schizophrenia was short-term efficacy, with the main aim of the clinician being to optimize the therapeutic response and improve positive symptoms. QoL was ranked fifth by the respondents after tolerability/safety, global functioning, and adherence to treatment. The lower rating for this aspect may have been because respondents' main focus was 
on shorter term, rather than long-term outcomes. QoL was interpreted in various ways, and was perceived mostly (58\%) as "relationships/social functioning". Of note, functioning was viewed in terms of the social and relationships aspects by the majority of respondents, but another $47 \%$ defined QoL as "recovered functioning/autonomy". The fact that other factors such as performance at work or school were less valued by psychiatrists may have been due to the high number of patients with schizophrenia who are unable to work or attend school. In addition, restoration of full functioning is necessary for performance at work and school, and therefore, the high rating for functional outcomes could also include job or study performance. The knowledge of scales to assess QoL was high among psychiatrists, and 76\% of them were familiar with QLS, which is a widely used tool in schizophrenia patients. ${ }^{41}$

QoL and functioning were considered important for assessing the success of therapy in patients with schizophrenia, including in patients receiving LAI antipsychotics. Assuming equal efficacy of agents, $47 \%$ and $40 \%$ of the respondents considered assessment of QoL and global functioning, respectively, as very important for assessing the success of therapy in patients with schizophrenia. Similarly, in patients receiving LAI antipsychotics, 40\% of psychiatrists considered these factors very important for the success of therapy. Several other studies cite factors such as psychosocial functioning and QoL as crucial to the definition of treatment success, ${ }^{37-39}$ and this concept is in line with the current treatment guidelines that recommend improvement of QoL and functioning as primary goals of long-term therapy. ${ }^{5}$

The types of patients most likely to be prescribed LAI antipsychotics according to our survey were "patients with low adherence in which oral therapy has failed". This concurs with data showing that poor adherence to oral antipsychotics is perceived as the most common cause of relapse in schizophrenia and that the use of LAI antipsychotics results in improved QoL and functioning. ${ }^{28}$ In addition, the fact that $77 \%$ of psychiatrists were unlikely to prescribe LAI antipsychotics to patients with no other treatment options (as shown by the low scores for this parameter) is positive and suggests that the current paradigm is changing - that is, psychiatrists are not limiting LAI only to those patients who have no other possibilities, but are considering them at different phases of treatment. Despite this, guidelines for use of LAI antipsychotics are conflicting. The American Psychiatric Association (APA) guidelines provide direction on the clinical evaluation of patients while on antipsychotic treatment in the acute, stabilization and maintenance phases. ${ }^{5}$ APA guidelines state that:

If a long-acting injectable medication is indicated, the oral form of the same medication [...] is the logical choice for initial treatment during the acute phase. ${ }^{5}$

It is also stated that LAI antipsychotics may improve adherence and outcomes in patients prone to poor adherence to drug regimens in any phase. ${ }^{5}$ Earlier guidelines recommended that depot/LAI antipsychotics should be considered for "any patients with schizophrenia for whom long-term treatment is indicated". ${ }^{42,43}$ The UK National Institute for Clinical Excellence guidelines state that LAI antipsychotics should be offered to all patients following an acute episode for long-term treatment, particularly in patients who need to avoid covert nonadherence. ${ }^{44}$ Recent publications have advocated offering LAI antipsychotics more widely, and most commentators emphasize the importance of offering LAI antipsychotics as part of a shared decision-making approach. ${ }^{42,45-48}$ Some guidelines, eg, the French Association for Biological Psychiatry and Neuropsychopharmacology guidelines, recommend that LAI antipsychotics should be considered as first-line therapy in any patient requiring long-term antipsychotic therapy. ${ }^{47}$ While Italian experience with LAI antipsychotics in patients with first-episode schizophrenia is limited, it has been suggested that the potential of these agents in early disease is promising and should be examined further. ${ }^{42}$ Data from Finland suggest that adherence to initial antipsychotic therapy in the first 60 days after discharge from first schizophrenia-related hospitalization is poor and that use of LAI rather than oral agents significantly reduces the risk of rehospitalization. ${ }^{49}$ The potential utility of LAI antipsychotics for early treatment of schizophrenia has also been highlighted by other authors. ${ }^{46,48}$

Previous studies to identify factors influencing physicians' choice of antipsychotic drug therapy in the treatment of patients with schizophrenia are limited; one prospective, naturalistic study investigated a range of variables and suggested that side effects and severity of positive symptoms were the main drivers of the choice of antipsychotic agent. ${ }^{50}$

QoL assessment scales are numerous and not standardized in schizophrenia trials or in the clinical setting. This survey showed that although psychiatrists know about QoL assessment scales and consider QoL assessment important, they confuse QoL with the factors that influence it (eg, interpersonal relationships), indicating a lack of consensus. The finding that most (67\%) psychiatrists use one of the available scales (QLS) is encouraging; however, 
recent research has shown that more specific disease-related QoL assessment scales (eg, WHOQOL-BREF) may be more reliable, useful, and valid than less specific QoL scales in the assessment of people with schizophrenia. ${ }^{24}$ The current survey revealed a wide range of opinions and practices within Italy, emphasizing the need for more research to elucidate the reasons for this and to determine ways to further standardize/ optimize the management of patients with schizophrenia, including QoL assessment. A recent review of QoL scales in schizophrenia also concluded that more research is required to reach a consensus on QoL measurement and its utility in predicting outcomes with different treatments in schizophrenia patients. ${ }^{41}$ Further research would also help determine the types of patients most likely to benefit from LAI antipsychotic therapy.

While the use of a large and representative sample of clinicians that covered a cross-section of psychiatric services can be considered a strength of this survey, there are also several limitations; mostly, those associated with the use of a survey. These include the method of selecting participants, the method of response (which may have increased or decreased the likelihood of responses), and responder bias (ie, those responding may be more likely to respond positively).

This survey, which is the first to solicit the opinions of psychiatrists on the use of QoL and other factors for evaluating the success of antipsychotic therapy, showed that, overall, psychiatrists considered efficacy and tolerability to be the most important factors for evaluating therapy in patients with schizophrenia. Another important factor for evaluating the success of antipsychotic therapy was improvement in QoL, which was perceived by the majority of respondents as "relationships/social functioning" and "recovered functioning/autonomy". The assessment of QoL and other subjective functional outcomes is important in the acute and long-term management of schizophrenia to identify treatments that can help patients lead a more fulfilling and satisfying life as a result of effective treatment of their disease. Novel LAI antipsychotic therapies have been shown to have a better balance between efficacy and safety, and to improve QoL due to improved adherence versus oral therapies. ${ }^{51}$ The survey showed that a range of QoL scales are used to define and assess QoL, and psychiatrists answering the survey thought that QoL assessment was important in assessing treatment response in patients with schizophrenia, including in patients treated with LAI antipsychotics. LAI antipsychotics were seen as an appropriate option for schizophrenia patients with low adherence for whom oral therapy had failed, and in those poorly controlled on oral therapy. More data are required to achieve consensus on which assessment measures, including QoL definitions and scales, are the most helpful in guiding management of schizophrenia and use of LAI antipsychotic therapy to improve overall long-term outcome.

\section{Key points}

- A survey was conducted to identify the primary drivers for treatment choice and the variables by which psychiatrists in Italy evaluate the success of antipsychotic therapy in general, and LAI in particular, in patients with schizophrenia.

- The most common factor influencing success of antipsychotic therapy in patients with schizophrenia according to psychiatrist was short-term efficacy ( $75 \%$ of responses), followed by tolerability (45\%), then global functioning (24\%), and QoL (17\%) with the main aim of the clinician being to optimize the therapeutic response and improve positive symptoms.

- LAI antipsychotics were most often used to facilitate regular treatment monitoring (49\%), and $41 \%$ of psychiatrists thought that patients with low adherence who had failed oral therapy were well-suited for LAI antipsychotics.

- Only 4\% of psychiatrics saw LAI antipsychotics as appropriate for patients without other therapeutic options.

\section{Acknowledgment}

Mary Hines of Springer Healthcare Communications provided medical writing support funded by Lundbeck and Otsuka Pharmaceutical Italy Srl.

\section{Disclosure}

This survey and paper was supported by Lundbeck and Otsuka Pharmaceutical Italy Srl.

AdB has been the recipient of preclinical research grants from Lundbeck, Otsuka, and Janssen; has been involved in Advisory Boards for Lundbeck, Roche, and Janssen; and has received honoraria as a speaker for educational activities funded by unrestricted grants from Chiesi, Janssen, Lundbeck, Otsuka, Roche, Astra Zeneca, and Bristol Myers Squibb.

$\mathrm{AF}$ has received research grants and/or has been a speaker and/or a consultant for Angelini, Astra Zeneca, Bristol-Myers Squibb, Boehringer Ingelheim, Eli Lilly, Janssen, Lundbeck, Novartis, Otsuka, Pfizer, and Roche. 
$\mathrm{CV}$ has been a speaker and/or consultant for Angelini, Astra Zeneca, Bristol-Myers Squibb, Ely Lilly, Janssen, Lundbeck, Otsuka, and Pfizer.

MV has been a speaker and/or consultant for Astra Zeneca, Bristol Myers Squibb, Chiesi, Janssen, Lilly, Lundbeck, Otsuka, and Pfizer.

The authors report no other conflicts of interest in this work.

\section{References}

1. Tandon R, Nasrallah HA, Keshavan MS. Schizophrenia, "just the facts" 4. Clinical features and conceptualization. Schizophr Res. 2009; 110(1-3):1-23.

2. American Psychiatric Association. Diagnostic and Statistical Manual of Mental Disorders. 5th ed. Arlington, VA: American Psychiatric Association; 2013.

3. Tandon R, Nasrallah HA, Keshavan MS. Schizophrenia, "just the facts" 5. Treatment and prevention. Past, present, and future. Schizophr Res. 2010;122(1-3):1-23.

4. van Os J, Kapur S. Schizophrenia. Lancet. 2009;374(9690):635-645.

5. Lehman AF, Lieberman JA, Dixon LB, et al. Practice guideline for the treatment of patients with schizophrenia, second edition: work group on schizophrenia [Guidelines] 2004. Available from: http:/ psychiatryonline.org/pb/assets/raw/sitewide/practice_guidelines/ guidelines/schizophrenia.pdf. Accessed April 7, 2015.

6. Thibaut F. Acute treatment of schizophrenia: introduction to the Word Federation of Societies of Biological Psychiatry guidelines. Psychiatr Dапиь. 2014;26(1):2-11.

7. van Os J, Kapur S. Psychosis: from diagnosis to syndrome. Ned Tijdschr Geneeskd. 2010;154:A1874. Dutch.

8. Hasan A, Falkai P, Wobrock T, et al. World Federation of Societies of Biological Psychiatry (WFSBP) guidelines for biological treatment of schizophrenia, part 2: update 2012 on the long-term treatment of schizophrenia and management of antipsychotic-induced side effects. World J Biol Psychiatry. 2013;14(1):2-44.

9. Harvey PD, Velligan DI, Bellack AS. Performance-based measures of functional skills: usefulness in clinical treatment studies. Schizophr Bull. 2007;33(5):1138-1148.

10. Loga-Zec S, Loga S. Antipsychotics and the quality of life of schizophrenic patients. Psychiatr Danub. 2010;22(4):495-497.

11. The WHOQOL Group. The World Health Organization Quality of Life Assessment (WHOQOL): development and general psychometric properties. Soc Sci Med. 1998;46(12):1569-1585.

12. Cella DF. Measuring quality of life in palliative care. Semin Oncol. 1995;22(2 Suppl 3):73-81.

13. Schipper H, Clinch JJ, Olweny CL. Quality of life studies: definitions and conceptual issues. In: Spilker B, editor. Quality of Life and Pharmacoeconomics in Clinical Trials. Philadelphia, PA: Lippincott-Raven; 1996:11-23.

14. Leidy NK. Functional status and the forward progress of merry-go-rounds: toward a coherent analytical framework. Nurs Res. 1994;43(4):196-202.

15. de Araujo AA, de Araujo Dantas D, do Nascimento GG, et al. Quality of life in patients with schizophrenia: the impact of socio-economic factors and adverse effects of atypical antipsychotics drugs. Psychiatr $Q$. 2014;85(3):357-367.

16. Kwon JS, Choi JS. Social functioning and quality of life as measures of effectiveness in the treatment of schizophrenia. World Psychiatry. 2009;8(1):35-36.

17. Yamauchi K, Aki H, Tomotake M, et al. Predictors of subjective and objective quality of life in outpatients with schizophrenia. Psychiatry Clin Neurosci. 2008;62(4):404-411.

18. Naber D, Vita A. Tools for measuring clinical effectiveness. Eur Neuropsychopharmacol. 2004;14(Suppl 4):S435-S444.
19. Franz M, Fritz M, Gallhofer B, Meyer T. QLiS - development of a schizophrenia-specific quality-of-life scale. Health Qual Life Outcomes. 2012;10:61

20. Russo J, Trujillo CA, Wingerson D, et al. The MOS 36-item short form health survey: reliability, validity, and preliminary findings in schizophrenic outpatients. Med Care. 1998;36(5):752-756.

21. Montejo AL, Lauffer JC, Cuervo J, et al. Validation of a specific measure to assess health-related quality of life in patients with schizophrenia and bipolar disorder: the 'Tolerability and quality of life' (TOOL) questionnaire. Ann Gen Psychiatry. 2011;10:6.

22. Vothknecht S, Meijer C, Zwinderman A, et al. Psychometric evaluation of the Subjective Well-being Under Neuroleptic Treatment Scale (SWN) in patients with schizophrenia, their relatives and controls. Psychiatry Res. 2013;206(1):62-67.

23. Riedel M, Spellmann I, Schennach-Wolff R, Obermeier M, Musil R. The RSM-scale: a pilot study on a new specific scale for self- and observer-rated quality of life in patients with schizophrenia. Qual Life Res. 2011;20(2):263-272.

24. Su CT, Ng HS, Yang AL, Lin CY. Psychometric evaluation of the Short Form 36 Health Survey (SF-36) and the World Health Organization Quality of Life Scale Brief Version (WHOQOLBREF) for patients with schizophrenia. Psychol Assess. 2014;26(3): 980-989.

25. Ascher-Svanum H, Faries DE, Zhu B, Ernst FR, Swartz MS, Swanson JW. Medication adherence and long-term functional outcomes in the treatment of schizophrenia in usual care. J Clin Psychiatry. 2006;67(3):453-460.

26. Haddad PM, Brain C, Scott J. Nonadherence with antipsychotic medication in schizophrenia: challenges and management strategies. Patient Relat Outcome Meas. 2014;5:43-62.

27. Bera RB. Patient outcomes within schizophrenia treatment: a look at the role of long-acting injectable antipsychotics. J Clin Psychiatry. 2014;75(Suppl 2):30-33.

28. Kaplan G, Casoy J, Zummo J. Impact of long-acting injectable antipsychotics on medication adherence and clinical, functional, and economic outcomes of schizophrenia. Patient Prefer Adherence. 2013;7: $1171-1180$.

29. Takeuchi H, Suzuki T, Uchida H, Watanabe K, Mimura M. Antipsychotic treatment for schizophrenia in the maintenance phase: a systematic review of the guidelines and algorithms. Schizophr Res. 2012; 134(2-3):219-225.

30. Keshavan MS, Roberts M, Wittmann D. Guidelines for clinical treatment of early course schizophrenia. Curr Psychiatry Rep. 2006;8(4):329-334.

31. Chugh PK, Rehan HS, Unni KE, Sah RK. Predictive value of symptoms for quality of life in first-episode schizophrenia. Nord J Psychiatry. 2013; 67(3):153-158.

32. Zouari L, Thabet JB, Elloumi Z, Elleuch M, Zouari N, Maalej M. Quality of life in patients with schizophrenia: a study of 100 cases. Encephale. 2012;38(2):111-117. French.

33. Fujimaki K, Takahashi T, Morinobu S. Association of typical versus atypical antipsychotics with symptoms and quality of life in schizophrenia. PLoS One. 2012;7(5):e37087.

34. Awad AG, Voruganti LN. Quality of life and new antipsychotics in schizophrenia. Are patients better off? Int J Soc Psychiatry. 1999; 45(4):268-275.

35. Barkhof E, Meijer CJ, de Sonneville LM, Linszen DH, de Haan L. Interventions to improve adherence to antipsychotic medication in patients with schizophrenia - a review of the past decade. Eur Psychiatry. 2012; 27(1):9-18.

36. Osborne RH, Dalton A, Hertel J, Schrover R, Smith DK. Healthrelated quality of life advantage of long-acting injectable antipsychotic treatment for schizophrenia: a time trade-off study. Health Qual Life Outcomes. 2012;10:35.

37. Buckley PF. Factors that influence treatment success in schizophrenia. J Clin Psychiatry. 2008;69(Suppl 3):4-10.

38. Collins EJ, Hogan TP, Desai H. Measurement of therapeutic response in schizophrenia. A critical survey. Schizophr Res. 1991;5(3):249-253. 
39. Juckel G, Morosini PL. The new approach: psychosocial functioning as a necessary outcome criterion for therapeutic success in schizophrenia. Curr Opin Psychiatry. 2008;21(6):630-639.

40. Kane JM. Improving patient outcomes in schizophrenia: achieving remission, preventing relapse, and measuring success. J Clin Psychiatry. 2013;74(9):e18.

41. Karow A, Wittmann L, Schottle D, Schafer I, Lambert M. The assessment of quality of life in clinical practice in patients with schizophrenia. Dialogues Clin Neurosci. 2014;16(2):185-195.

42. Altamura AC, Aguglia E, Bassi M, et al. Rethinking the role of long-acting atypical antipsychotics in the community setting. Int Clin Psychopharmacol. 2012;27(6):336-349.

43. Kane JM, Aguglia E, Altamura AC, et al. Guidelines for depot antipsychotic treatment in schizophrenia. European Neuropsychopharmacology Consensus Conference in Siena, Italy. Eur Neuropsychopharmacol. 1998; 8(1):55-66.

44. National Institute for Health and Care Excellence [NICE]. Schizophrenia: Core Interventions in the Treatment and Management of Schizophrenia in Primary and Secondary Care. NICE clinical guideline 82. Leicester, UK: British Psychological Society; 2009.

45. Kirschner M, Theodoridou A, Fusar-Poli P, Kaiser S, Jager M. Patients' and clinicians' attitude towards long-acting depot antipsychotics in subjects with a first episode of psychosis. Ther Adv Psychopharmacol. 2013; 3(2):89-99.
46. Parellada E, Velligan DI, Emsley R, Kissling W. Long-acting injectable antipsychotics in first-episode schizophrenia. Schizophr Res Treatment. 2012;2012:318535.

47. Llorca PM, Abbar M, Courtet P, Guillaume S, Lancrenon S, Samalin L. Guidelines for the use and management of long-acting injectable antipsychotics in serious mental illness. BMC Psychiatry. 2013;13:340.

48. Brissos S, Veguilla MR, Taylor D, Balanza-Martinez V. The role of long-acting injectable antipsychotics in schizophrenia: a critical appraisal. Ther Adv Psychopharmacol. 2014;4(5):198-219.

49. Tiihonen J, Haukka J, Taylor M, Haddad PM, Patel MX, Korhonen P. A nationwide cohort study of oral and depot antipsychotics after first hospitalization for schizophrenia. Am J Psychiatry. 2011;168(6): 603-609.

50. Edlinger M, Hofer A, Rettenbacher MA, et al. Factors influencing the choice of new generation antipsychotic medication in the treatment of patients with schizophrenia. Schizophr Res. 2009;113(2-3):246-251.

51. Agid O, Foussias G, Remington G. Long-acting injectable antipsychotics in the treatment of schizophrenia: their role in relapse prevention. Expert Opin Pharmacother. 2010;11(14):2301-2317.

\section{Publish your work in this journal}

Neuropsychiatric Disease and Treatment is an international, peerreviewed journal of clinical therapeutics and pharmacology focusing on concise rapid reporting of clinical or pre-clinical studies on a range of neuropsychiatric and neurological disorders. This journal is indexed on PubMed Central, the 'PsycINFO' database and CAS,

\section{Dovepress}

and is the official journal of The International Neuropsychiatric Association (INA). The manuscript management system is completely online and includes a very quick and fair peer-review system, which is all easy to use. Visit http://www.dovepress.com/testimonials.php to read real quotes from published authors. 